\section{Using pathology data to evaluate surgical backlogs: considerations for resource planning}

Wang and colleagues provided valuable insight into the impact of coronavirus disease 2019 (COVID-19), estimating a backlog of 148364 surgeries in Ontario from March to June 2020. ${ }^{1}$ Expanding on the issues they raised, we would like to highlight important additional considerations.

We reviewed surgical pathology and Cancer Care Ontario summary data from the London Health Sciences Centre and St. Joseph's Health Care in London, Ontario. In April 2020, compared with April 2019 , the total number of specimens received decreased by $67.5 \%$ (2820 v. 8668), and resection specimens decreased by $51.4 \%$ (524 v. 1078). Cancer Care Ontario report submissions, largely reflecting newly staged cancer cases, decreased by $30.5 \%$ (169 v. 243). The relatively modest drop in resections and Cancer Care Ontario submissions relative to total specimens likely reflects efforts to prioritize cancer surgeries.

In the period from April to June 2020, compared with 2019, the gap decreased as surgeries resumed. Total specimens were reduced by $47.4 \%$ (13 842 v. 26 303), compared with $30.9 \%$ for resections (2304 v. 3335 ) and $11.3 \%$ for Cancer Care Ontario submissions (643 v. 725). Of note, reductions in Cancer Care Ontario submissions varied between disease sites. Although volumes were comparable for breast cancer (170 v. 171) and colon cancer (73 v. 76), there were drops of $71.6 \%$ (19 v. 67) and 53.2\% (29 v. 62) for lung and prostate cancers, respectively. These differences may be a result of triaging based on patient, clinical, safety and resource factors. ${ }^{2}$ We suspect that regional variations and surgery reductions have limited cancer procedures to high-risk cases and may have resulted in underserviced areas.

It is important that biopsy services are maintained, to identify patients who require prompt treatment. Depending on the disease and site, biopsies may be performed in a variety of settings, such as endoscopy suites, operating rooms and clinics. As such, biopsy volumes in April to June were variably decreased between disease sites, with colorectal biopsies down $63.7 \%$ (2013 v. 5553), compared with $24.4 \%$ for breast biopsies (374 v. 495) and $23.9 \%$ for prostate biopsies (730 v. 959).

Service reductions owing to COVID-19 resulted in decreased surgical resection, cancer reporting and biopsy volumes. Variable effects between disease sites may highlight gaps in care; resource planning to address such gaps should ensure patients have fair and equitable access to health care resources. The impact of the surgical backlog also extends beyond operating rooms and the health care sector, including pressures on primary care, long-term care homes and ability to return to work. We recognize that COVID-19 will continue to affect surgical activity, particularly when pressures on the acute sector grow from COVID-19-related outbreaks and admissions. As system leaders grapple with hospital capacity and demand, we encourage that system-wide risk assessments be undertaken. Prompt and targeted investments to expand diagnostic and surgical activity through all possible care settings will likely pay long-term dividends. Delays in addressing this challenge until after the COVID-19 pandemic is over not only allow for symptoms to escalate, but will also be costly.

\section{Christopher Tran MD MSc}

Resident physician in anatomical pathology, Department of Pathology and Laboratory Medicine, Western University; London Health Sciences Centre, London, Ont.

\section{Mike Kadour PhD}

Director for pathology and laboratory medicine, Department of Pathology and Laboratory Medicine, Western University; London Health Sciences Centre, London, Ont.

\section{Matthew J. Cecchini MD PhD}

Assistant professor and anatomical pathologist, Department of Pathology and Laboratory Medicine, Western University; London Health Sciences Centre, London, Ont.

\section{Ken A. Leslie MD}

Professor, chair/chief, and general surgeon, Department of Surgery, Western University; London Health Sciences Centre, London, Ont.

\section{David K. Driman MBChB}

Professor and anatomical pathologist, Department of Pathology and Laboratory Medicine, Western University; London Health Sciences Centre, London, Ont.

Cite as: CMAJ 2021 March 8;193:E343. doi: $10.1503 / \mathrm{cmaj} .77883$

\section{References}

1. Wang J, Vahid S, Eberg M, et al. Clearing the surgical backlog caused by COVID-19 in Ontario: a time series modelling study. CMAJ 2020;192: E1347-56.

2. COVID-19 supplemental clinical guidance for patients with cancer. Toronto: Ontario Health; 2020. Available: www.ontariohealth.ca/sites/ ontariohealth/files/2020-04/Ontario\%20Health\% 20Cancer\%20Care\%200ntario\%20COVID-19\%20 Supplemental\%20Clinical\%20Guidance $\% 20$ for $\%$ 20Patients\%20with\%20Cancer_29Mar20\%20PDF. pdf (accessed 2020 Nov. 23).

\section{Competing interests: None declared.}

Content licence: This is an Open Access article distributed in accordance with the terms of the Creative Commons Attribution (CC BY-NC-ND 4.0) licence, which permits use, distribution and reproduction in any medium, provided that the original publication is properly cited, the use is noncommercial (i.e., research or educational use), and no modifications or adaptations are made. See: https://creativecommons.org/ licenses/by-nc-nd/4.0/ 